\title{
When patients and surgeons disagree about surgical outcome: investigating patient factors and chart note communication
}

\author{
Carolyn E. Schwartz ${ }^{1,2^{*}}$, Armon Ayandeh ${ }^{1}$ and Joel A. Finkelstein ${ }^{3}$
}

\begin{abstract}
Objective: Effective physician-patient communication is a critical component of a clinical practice and in order to achieve optimal patient outcomes. We aimed to investigate indirect effects of physician-patient communication by examining the relationship between a physician-patient mismatch in perceived outcomes and content in the medical record's clinical note. We compared patient records whose perceived subjective assessment of surgery outcomes agreed or disagreed with the surgeon's perception of that outcome (Subjective Disagreement).
\end{abstract}

Methods: This study included 172 spine surgery patients at a teaching hospital. Patient-reported outcomes included the Oswestry Disability Index; the Short-Form 36; and a Visual Analogue Scale items for leg and back pain. We content-analyzed the clinical note in the medical record, and used logistic regression to evaluate predictors of Subjective Disagreement ( $n=41$ disagreed vs. 131 agreed).

Results: Patient and surgeon agreed in $76 \%$ of cases and disagreed in $24 \%$ of cases. Patients who assessed their outcome worse than their surgeons tended to be less educated and involved in litigation. They also tended to report worsened mental health and leg pain. Content analysis revealed group differences in surgeon communication patterns in the chart notes related to how symptom change was emphasized, how follow-up was described, and a specific word reference. Specifically, disagreement was predicted by using "much" to emphasize the findings and noting long-term prognosis. Agreement was predicted by use of positive emphasis terms, having an "as-needed" follow-up plan, and using "happy" in the chart note.

Conclusion: The nature of measuring outcomes of surgery is based on patient perception. In surgeon-patient perspective mismatches, patient factors may serve as barriers to improvement. Worsened change on patient-reported mental health may be an independent factor which colors the patient's general perceptions. This aspect of treatment may be missed by the spine surgeon. Chart note communication styles reflect the subjective disagreement. Investigating and/ or treating mental health deterioration may be valuable in resolving this mismatch and for overall outcome.

Keywords: Communication, Patient-reported outcomes, Clinical outcomes, Mismatch, Response shift, Medical record, Disability

\section{Introduction}

The art and science of medicine has needed to evolve in response to improvements in technology, patient expectations, and an increasingly litigious world. The training of future physicians has likewise evolved, and curricula sanctioned by governing bodies have helped maintain a

\footnotetext{
* Correspondence: carolyn.schwartz@deltaquest.org

'DeltaQuest Foundation, Inc., 31 Mitchell Road, Concord, MA 01742, USA

${ }^{2}$ Departments of Medicine and Orthopaedic Surgery, Tufts University Medical School, Boston, MA, USA

Full list of author information is available at the end of the article
}

high standard of care. One such body, the Canadian Medical Education Directives for Specialists (CanMEDS) $[1,2]$, is a competency-based framework that has been adopted to guide training programs and evaluations of physicians and specialists. The communicator role is a particularly essential aspect of this framework for establishing rapport and trust, formulating a diagnosis, delivering information, striving for mutual understanding, and facilitating a shared plan of care. Effective communication is critical for optimal patient outcomes. 
Central to effective communication is being able to convey orally to the patient realistic expectations about surgical outcome, as well as effective written information about a medical encounter [3]. The purpose of this study was to examine predictors of physician-patient mismatch in perceived outcomes of spine surgery. We compared patient records whose perceived subjective assessment of surgery outcomes agreed or disagreed with the surgeon's perception of that outcome, and investigated demographic factors, patient-reported outcomes, and the clinical chart note content in the medical record.

\section{Materials and methods}

\section{Sample and design}

This prospective study included patients who had undergone one or two level decompression surgery; (discectomy or laminectomy) with leg-dominant pain. Multilevel decompressions and fusion procedures that require longer rehabilitation until maximum recovery were excluded. Excluding patients with more complicated operations was done to create a homogenous group of patients Study participants were consecutively recruited from the practices of three spine surgeons at a major teaching hospital in Canada. As standard protocol, surgeons engaged all patients in a transparent discussion about what to expect from the surgery. The surgeons explained what the surgery will improve and will not improve, i.e. leg pain will be better, back pain may or may not. Patients provided baseline data pre-surgically, and follow-up data post-surgery. The study protocol was approved by the Sunnybrook Health Sciences Centre Research Ethics Board, and all participants provided written informed consent. Participants completed self-report questionnaires pre-surgery and at first follow-up.

\section{Measures}

Three categories of measures were used in this study. Demographic characteristics were collected from the patients, including age, gender, duration of symptoms, employment status (working at present, retired, student, homemaker), smoking status (i.e., current smoker or not), and associated co-morbid health conditions and other musculoskeletal conditions. We also tracked having an incentive not to work. This variable was characterized as involvement in compensation or litigation that would serve as an incentive not to experience symptom improvement over time (e.g., currently on disability or worker's compensation, or involved in litigation related to their illness or injury.

Standardized spine outcome measures were collected in this study: (1) the generic Short-Form-36 v1 (SF36v1) [4] comprising eight domains assessing evaluative functional health, with higher scores reflecting better functional health; (2) two Likert-scaled visual analogue scale (VAS) items measuring back and leg pain on a 100-point scale, with higher scores indicating worse pain [2]; (3) The 10-item disease specific Oswestry Disability Index (ODI) [1] measuring perceived pain during activities of daily living.

Clinical chart notes were also utilized in this study. These notes included data from the neurological examination (e.g., straight leg raising, numbness, strength, walking distance); as well as the recorded subjective assessment of surgical outcome from patient and surgeon. Patients were asked how they would characterize their surgical outcome (poor, moderate, excellent). Surgeons were asked to note in the chart how they would characterize the patient's outcome based on the objective examination, as well as their understanding of the patient's change in function. Surgeons categorized this understanding as not improved, not fully improved, or fully improved. The chart note also provided information on whether there were complications from the surgery; and reported symptoms of leg or back pain. Additionally, written summaries of the patient's follow-up appointment were captured verbatim. These included documentation for the patient's medical record as well as communications to other health care providers.

\section{Content analysis}

Text from the clinical chart notes were content analyzed using QSR NVIVO 10 [5]. Two independent raters read and coded all chart notes for terms or concepts that were identified after coding an initial 100 patients (see Additional file 1 for complete listing and explanation of nodes). After all records were coded by both raters, inter-rater reliability was computed using the kappa coefficient [6]. It was greater than $90 \%$ on most nodes. Adjudication then took place such that all differences in codes were discussed to determine the most appropriate coding for the record. This process resulted in $100 \%$ inter-rater reliability.

\section{Statistical analysis}

Patients were characterized on the basis of whether their subjective assessment of surgery outcome was similar or disagreed with the surgeon's assessment. We then used this grouping variable (Subjective Agreement vs Subjective Disagreement) as the dependent variable in a series of hierarchical logistic regression analyses. We began with univariate regressions within a class of variables (i.e., demographic, patient-reported outcome, clinicalchart nodes). We then computed multivariate models within a class of variables. We did not combine the three classes of variables into a single multivariate model, due to sample size constraints and the resulting limited power. We thus present the results of the three sets of models in terms of triangulating on the prediction of the grouping variable. The Type I error rate for the univariate 
models was $p<0.10$, and $p<0.05$ for the multivariable model, as per standard hierarchical modeling approaches. Stata 13 [7] was used for logistic regression modeling.

\section{Results}

Table 1 shows how the sample was characterized in terms of subjective assessment of surgical outcome. There were 130 patients $(76 \%)$ whose subjective assessment agreed with the surgeon's, one who thought the outcome was better than the surgeon did, and 41 (24\%) who thought they did worse than the surgeon thought. For the purpose of the subsequent analyses, we dropped the one outlier who thought $\mathrm{s} / \mathrm{he}$ did better than the surgeon thought because this person would have been qualitatively different than both those who agreed and those who disagreed with their surgeon's assessment.

The remaining 171 patients had a mean post-operative follow-up of 7.7 weeks $(S D=3.2)$. The procedures which were the basis of the study would be expected to result in significant recovery by this time point as the operative morbidity rates are generally low. Table 2 provides the descriptive statistics on the demographics, comorbidities, and baseline patient-reported outcome scores for the 171 people who were retained in the analysis.

\section{Demographic predictors of subjective disagreement}

Multivariate logistic models suggested that having a subjective assessment of outcome that was worse than their surgeon's was more likely among people who had lower education $(\mathrm{OR}=0.20, p<0.01)$, and who had an incentive not to work (OR $=4.3, p<0.01)$ (Table 3). There was no impact of age, gender, comorbidities, or pre-surgical use of narcotic analgesic, or pre-surgical smoking (Appendix).

\section{Patient-reported outcome predictors of subjective disagreement}

Multivariate models suggested that patients whose subjective assessment of outcome was worse than their surgeons tended to report worsened mental health component scores on the SF-36, and worsened VAS leg pain (OR $=0.94$ and $1.03, p<0.05$ and 0.02 , respectively) (Table 4 ). There was

Table 1 Characterization of Patient-Surgeon Agreement on Surgery Outcome ${ }^{a}$

\begin{tabular}{lllll}
\hline & \multicolumn{3}{l}{ Patient's perspective } & \\
\cline { 2 - 4 } Surgeon's perspective & Poor & Moderate & Excellent & Total \\
\hline Not improved & 3 & $\mathbf{1}$ & 0 & 4 \\
Not fully improved & $\mathbf{9}$ & 2 & 0 & 11 \\
Fully improved & 0 & $\mathbf{3 2}$ & 125 & 157 \\
Total & 12 & 35 & 125 & 172 \\
\hline
\end{tabular}

Table 2 Sample Demographics

\begin{tabular}{ll}
\hline Variable & $N=171(\%$ or SD) \\
\hline Time points & $7.73(3.23)$ \\
$\quad$ Mean Weeks of Follow-up (SD) & \\
Gender: N (\%) & $80(46.78)$ \\
Male & $60(35.09)$ \\
Female & $31(18.13)$ \\
Missing & \\
Surgical Diagnosis ${ }^{a}: N(\%)$ & $97(56.73)$ \\
Disc Herniation & $69(40.35)$ \\
Spinal Stenosis & $29(16.96)$ \\
Spondylolithesis & $8(4.68)$ \\
Other &
\end{tabular}

Co-morbidities: N (\%)

Depression 14 (8.19)

Cardiac Conditions $\quad 11(6.43)$

Diabetes $13(7.60)$

Thyroid Conditions 5 (2.92)

Cancer $\quad 5(2.92)$

Pulmonary Conditions $\quad 4$ (2.08)

Stroke 2 (1.17)

Peripheral Neuropathy $2(1.17)$

Other 19 (11.11)

Education: $N(\%)$

Less than High School 12 (7.02)

Graduated From High School or GED 23 (13.45)

Some College or Technical School 28 (16.37)

Graduated from College $\quad 30$ (17.54)

Postgraduate School or Degree $\quad 39$ (22.81)

Missing

$39(22.81)$

Employment Status at Pre-surgical Baseline ${ }^{\mathrm{b}}$ : N (\%)

Working 56 (32.75)

On leave of absence $\quad 11(6.43)$

Unemployed 6 (3.51)

Retired $\quad 42(24.56)$

Disabled $17(9.94)$

Homemaker 6 (3.51)

Student $\quad 5(2.92)$

Other $\quad 15(8.77)$

Missing 17 (9.94)

Pain Medication Use at Pre-surgical Baseline: N (\%)

Use Narcotics 37 (21.64)

Current Smoker $\quad 39$ (22.81)

Age: Mean Years (SD) $\quad 51.95$ (16.59)

Range [20-84]

${ }^{a}$ Bolded values represent discrepancy groups 
Table 2 Sample Demographics (Continued)

\begin{tabular}{lc}
\hline Pre-surgical Baseline Patient-Reported Outcome Scores: Mean (SD) \\
SF-36 PCS & $32.68(8.19)$ \\
SF-36 MCS & $43.91(12.84)$ \\
VAS Back & $54.78(30.43)$ \\
VAS Leg & $65.36(26.45)$ \\
ODI & $24.58(10.96)$
\end{tabular}

${ }^{a} 6$ people had more than one diagnosis; 92 people had no diagnosis

$b_{3}$ people reported more than 1 category

no impact of change in VAS back pain, physical component score of the SF-36, or ODI (Table 4).

Clinical-chart node predictors of subjective disagreement Multivariate logistic models of clinical chart-note coded nodes revealed that when there was disagreement between the perceived outcomes, surgeons were more likely to use "much"-emphasis terms (e.g., much better, much different, much improved, much more, so much) and to discuss prognosis of presenting symptoms (e.g. any mention of how patients symptoms will change in the future), that is that they would improve with time $(\mathrm{OR}=5.5$ and 4.5, respectively; $p<0.02$ for both) (Table 5 and Fig. 1). Chart note predictors of patientsurgeon agreement included using positive language to emphasize the findings (e.g., absolutely, whatsoever, completely, extremely, very) $(\mathrm{OR}=0.20, p<0.02)$; having a follow-up plan that was open-ended (i.e., only if the patient presented with new or worsened symptoms) $(\mathrm{OR}=0.06, p<0.02)$, and the use of the term "happy" in the chart note $(\mathrm{OR}=0.15, p<0.04)$. There was no impact of factors such as re-engaging in activities of daily living, having an action plan, negative emphasis terms, symptoms, short follow-up plans, or other specific communication patterns coded (see Appendix for univariate analyses, and Additional file 1 for full listing of chart note codes).

\section{Discussion}

Our results suggest that patient-surgeon perspective mismatches may relate to layers of factors, beginning with patient characteristics, continuing through patientreported outcomes, and ending up as meta-messages (i.e., reading between the lines) transmitted in the medical record. The subtlety of these findings underscores the value of a qualitative analysis. Such patterns were detected only after careful coding and pattern analysis using a method that combined qualitative and quantitative techniques. This mixed-methods approach results in findings that would not be apparent from a simple reading of the text. They are only revealed by dint of careful data analysis.

Lower levels of education, which can reflect low health literacy, were predictive of disagreement with their surgeon. Being involved in any litigation related to their spinal disorder (i.e., worker's compensation, disability, or other litigation) also served as a risk factor for disagreement. Our findings underscore the potential bias in the self-report of patients in secondary-gain situations $[6,7]$, such as worker's compensation and litigation. Our data suggest that when people are in a situation where they benefit from not getting better, their answers to patientreported outcome questionnaires may not be valid. No measure, no matter how well it has demonstrated reliability and validity, can counteract the influence of secondary gain. Our findings are reminiscent of early work by Hayes and colleagues documenting that psychometric test results are unreliable among patients with nonorganic signs [8].

An unexpected finding was that the patient's reporting worsened mental health or worsened leg pain, (as opposed to no change in leg pain) after surgery were significant factors in subjective disagreement. It should be noted that the basis of the surgeon's assessment is on both objective and subjective grounds; including findings on the examination, notably the presence or absence of pain on straight leg-raising.

Our content analysis revealed subtle differences in how emphasis-language was used that differentiated patients whose subjective assessment differed from their surgeons. In addition to these differing ways of emphasizing their clinical findings, the chart text differed in how long-term follow-up was described. Whereas patients whose

Table 3 Logistic regression model of significant demographic factors predicting disagreement between doctor and patient

\begin{tabular}{|c|c|c|c|c|c|c|c|c|}
\hline & Odds Ratio & Std. Err. & z & $P$-value & $95 \%$ Conf. & Interval & $n$ & Pseudo r2 \\
\hline Demographic Factors Model & & & & $0.0058^{*}$ & & & 83 & 0.14 \\
\hline Education Bin & 0.20 & 0.13 & -2.57 & 0.01 & 0.060 & 0.685 & & \\
\hline NoWorklncentive & 4.31 & 2.40 & 2.61 & 0.009 & 1.44 & 12.87 & & \\
\hline \multicolumn{9}{|l|}{ Smoking } \\
\hline Never Smoked & Referent & & & & & & & \\
\hline Quit over a year ago & 0.30 & 0.19 & -1.85 & 0.064 & 0.083 & 1.073 & & \\
\hline Current Smoker & 0.24 & 0.18 & -1.87 & 0.061 & 0.053 & 1.071 & & \\
\hline
\end{tabular}

${ }^{*} P$-value specified for the model is based on a chi2 test, whereas individual item $p$-values are calculated from the $z$-statistic 
Table 4 Logistic regression model of significant patient-reported outcomes predicting disagreement between doctor and patient

\begin{tabular}{|c|c|c|c|c|c|c|c|c|}
\hline & Odds Ratio & Std. Err. & z & $P$-value & $95 \%$ Conf. & Interval & $n$ & Pseudo r2 \\
\hline Patient-reported Outcomes Model & & & & $0.0001^{*}$ & & & 84 & 0.24 \\
\hline VAS Back Change & 1.00 & 0.01 & 0.42 & 0.68 & 0.98 & 1.03 & & \\
\hline VAS Leg Change & 1.03 & 0.01 & 2.38 & 0.017 & 1.00 & 1.05 & & \\
\hline MCS Change & 0.94 & 0.03 & -2.00 & 0.046 & 0.88 & 1.00 & & \\
\hline PCS Change & 1.00 & 0.05 & 0.08 & 0.94 & 0.92 & 1.10 & & \\
\hline ODI Change & 1.02 & 0.04 & 0.41 & 0.68 & 0.94 & 1.10 & & \\
\hline
\end{tabular}

${ }^{*} P$-value specified for the model is based on a chi2 test, whereas individual item $p$-values are calculated from the $z$-statistic

subjective assessment agreed with their surgeons were more likely to have non-specific follow-up planned (i.e., on an as-needed basis), those who disagreed with their surgeons were more likely to have chart notes that suggested that symptoms would improve with time without mention of a specific plan for a medical encounter. Finally, those whose subjective assessment agreed with their surgeons were more likely to have the term "happy" in their medical record.

Since the clinical chart note is a largely codified document, there are many terms and content domains that must be mentioned. It is thus not surprising that many

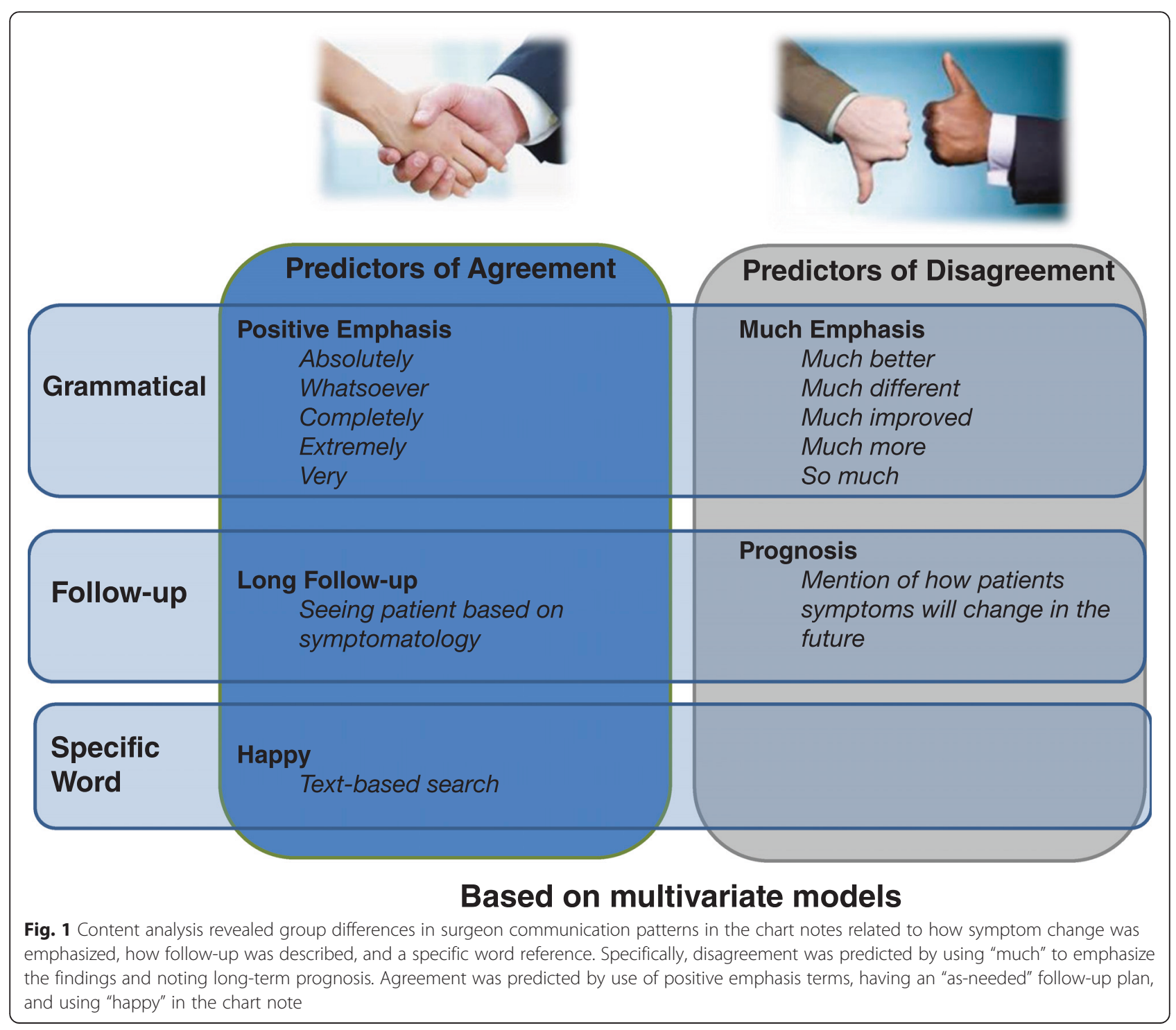


Table 5 Logistic regression model of significant clinical chart notes predicting disagreement between doctor and patient

\begin{tabular}{|c|c|c|c|c|c|c|c|c|}
\hline & Odds Ratio & Std. Err. & z & $P$-value & $95 \%$ Conf. & Interval & $n$ & Pseudo r2 \\
\hline Clinical Chart Notes Model & & & & $<0.0001^{*}$ & & & 171 & 0.3813 \\
\hline Positive Emphasis terms & 0.20 & 0.13 & -2.46 & 0.014 & 0.053 & 0.719 & & \\
\hline Much Emphasis terms & 5.51 & 3.71 & 2.54 & 0.011 & 1.47 & 20.59 & & \\
\hline All Emphasis terms & 0.41 & 0.27 & -1.34 & 0.18 & 0.11 & 1.51 & & \\
\hline All Juxtaposition terms & 1.17 & 0.57 & 0.31 & 0.75 & 0.45 & 3.05 & & \\
\hline All Analgesics & 2.19 & 1.57 & 1.09 & 0.28 & 0.54 & 8.92 & & \\
\hline Discomfort & 4.22 & 3.20 & 1.9 & 0.058 & 0.95 & 18.68 & & \\
\hline Prognosis & 4.46 & 2.65 & 2.51 & 0.012 & 1.39 & 14.32 & & \\
\hline Follow-up only if symptoms & 0.058 & 0.068 & -2.45 & 0.014 & 0.0060 & 0.57 & & \\
\hline Improve Stemmed & 1.24 & 0.67 & 0.39 & 0.70 & 0.43 & 3.57 & & \\
\hline Happy & 0.15 & 0.13 & -2.14 & 0.032 & 0.026 & 0.85 & & \\
\hline Patient Discharged & 3.53 & 3.85 & 1.16 & 0.25 & 0.42 & 29.96 & & \\
\hline
\end{tabular}

${ }^{*} P$-value specified for the model is based on a chi2 test, whereas individual item $p$-values are calculated from the $z$-statistic

relevant content areas did not differentiate the patient groups. For example, re-engagement in activities of daily living, having an action plan, and utilizing physical therapy are all expected aspects of surgical follow-up. These were not terms or content that differentiated our groups.

The clinical record may be saying more than the written word expresses, and it is possible the surgeon may be ill-prepared to appreciate the causes of the mismatch. The deterioration in mental health may be a harbinger of other personal or social factors in the patient's life. The worsening of mental health would not be an expected outcome from the surgery itself, but may be related to external influences (e.g., increased interpersonal conflict due to financial or marital strain related to being unwell). It may also reflect poor adaptation to the patient's new status quo. Mental health deterioration can lead to a negative coloring on all the subjective parameters of outcome, including leg pain, which was a predictor of subjective mismatch. Our findings suggest that measuring mental health status over time is not only important for understanding the patient's well-being, but may also help to elucidate subjective-mismatch situations. On the basis of change in patient-reported measures of mental health, an appropriate referral can be made.

The implications of our findings for improving clinical outcomes of spine surgery might focus on interventions that focus on improving health literacy and insight. Such an intervention might focus on adjusting patient expectations of surgical outcomes to be more consistent with the likely outcome, and on increasing their insight into the negative impact of litigation/ compensation on their health and well-being. The impact of such interventions on the eventual chart-note and surgeon-patient communication would be a useful path for future research.
The limitations of the present work should be noted. Whereas we chose a short follow-up time period that would be reflective of the pathology and treatment studied, a longer follow-up would be of more value except that the generic nature of patient-reported outcomes can introduce other biases due to changing life events that are unrelated to the surgery. There is also a possible bias introduced by missing data. Indeed, missing data issues restricted our ability to consider all classes of factors in one multivariate model because the sample size was substantially reduced when we did so. Further, our study included patients from a small number of surgeons (3) and from a country with notable socialized healthcare (Canada), both factors that may limit the generalizability of our findings.

Future work might continue our study's line of research by replicating its delineation of patterns associated with surgeon-patient mismatch using PROs, content analysis of clinical chart notes, and demographic factors along with collecting a information on nonorganic signs (e.g., the Waddell Nonorganic Signs Test [9]).

\section{Conclusions}

In summary, our findings underscore the multiple dimensions involved in surgeon-patient disagreement about subjective outcomes of spine surgery. In our study, this disagreement was apparent in about one quarter of the patients. Deterioration in the patient's mental health score was a predictor of subjective disagreement, a context which may color the overall perception of the patient. The surgeon should be mindful of this, and may be in a position to facilitate other forms of support for their patients. This problem is worthy of further research to further characterize risk factors, and to investigate approaches to intervene at multiple levels to prevent disagreement and improve overall satisfaction with and outcomes of spine surgery. 


\section{Appendix}

Table 6 Univariate Logistic Regression Model Results

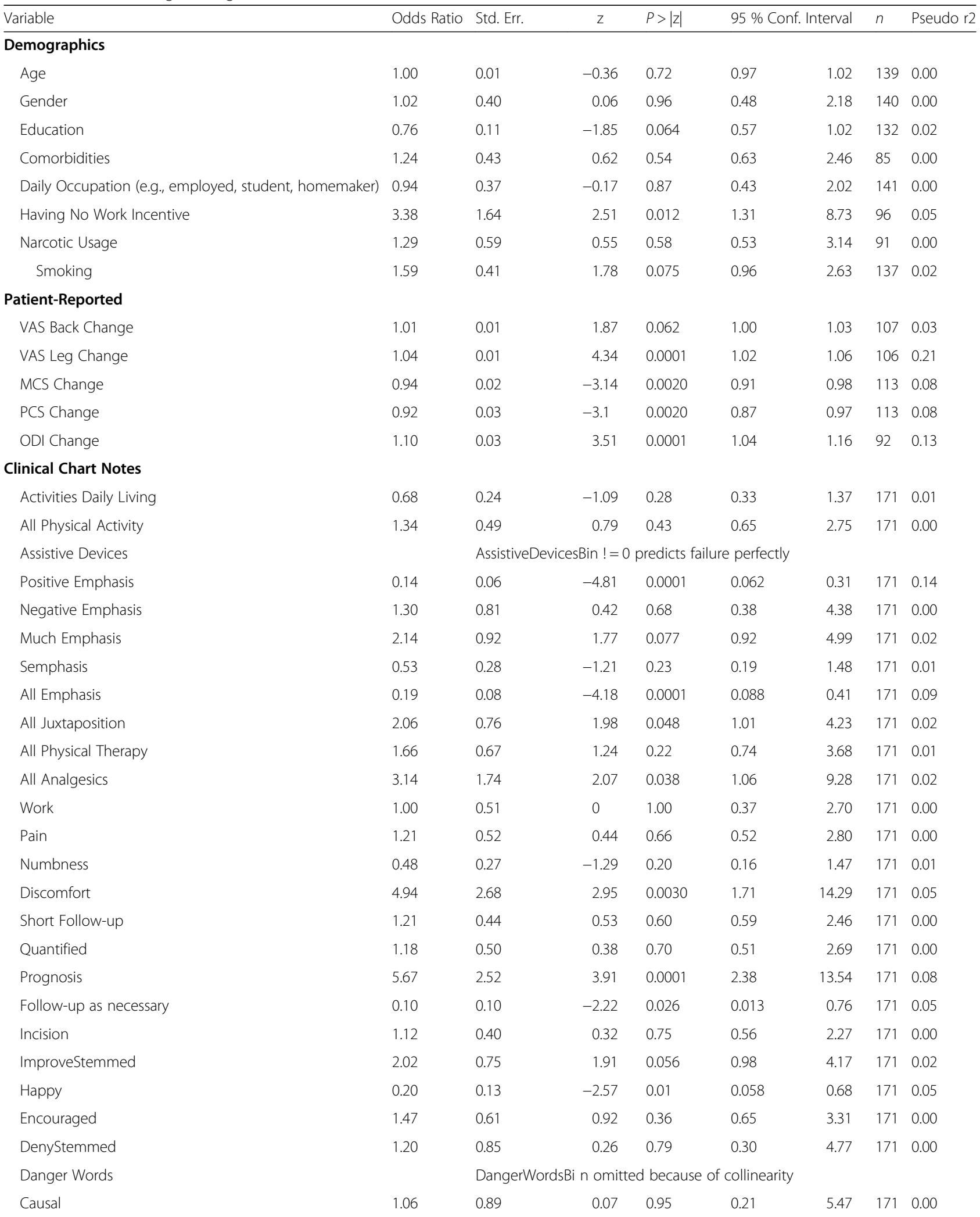


Table 6 Univariate Logistic Regression Model Results (Continued)

\begin{tabular}{|c|c|c|c|c|c|c|c|c|}
\hline Medium Follow-up & 0.91 & 0.43 & -0.2 & 0.84 & 0.36 & 2.30 & 171 & 0.00 \\
\hline No further follow-up & 5.05 & 4.71 & 1.74 & 0.082 & 0.81 & 31.35 & 171 & 0.02 \\
\hline Moving goal post & 3.28 & 3.34 & 1.17 & 0.24 & 0.45 & 24.07 & 171 & 0.01 \\
\hline Patient Non Pronoun & \multicolumn{8}{|c|}{ PatientNonPronounFinalBin $!=1$ predicts failure perfectly } \\
\hline Action Plan & 1.62 & 0.64 & 1.21 & 0.23 & 0.74 & 3.52 & 171 & 0.01 \\
\hline
\end{tabular}

\section{Additional file}

Additional file 1: Chart Note Node Codes Used in Content Analysis. (PDF $83 \mathrm{~kb}$ )

\section{Competing interest}

This work was not funded by any granting agency. The authors have no conflicts of interest to disclose. This work has been presented as an oral presentation at the International Society for Quality of Life Research in Berlin, Germany, October 2013; and as a poster presentation at the AO North America One AO Meeting in Las Vegas, Nevada, February 2015. None of the authors have competing interests in the manuscript.

\section{Authors' contributions}

CES and JAF conceived the study and participated in its design. AA and CES performed the statistical analysis. JAF and CES participated in coordination of the chart abstraction. CES and AA participated in the coding of the chart data. CES, JAF, and AA helped to draft the manuscript. All authors read and approved the final manuscript.

\section{Acknowledgements}

We gratefully acknowledge Albert Yee, M.D., and Michael Ford, M.D., for providing access to patients; Rebecca MacDonald for her assistance with chart abstraction; Kimberly Mulvehill for her assistance with coding chart notes; and Victoria Powell, M.P.H., for assistance with data management.

\section{Author details}

'DeltaQuest Foundation, Inc., 31 Mitchell Road, Concord, MA 01742, USA. ${ }^{2}$ Departments of Medicine and Orthopaedic Surgery, Tufts University Medical School, Boston, MA, USA. ${ }^{3}$ Division of Orthopaedics, Sunnybrook Health Sciences Center and the University of Toronto, 2075 Bayview Avenue, Room MG361, Toronto, ON, Canada.

Received: 16 April 2015 Accepted: 10 September 2015

Published online: 29 September 2015

\section{References}

1. Chou S, Cole G, McLaughlin K, Lockye J. CanMEDS evaluation in Canadian postgraduate training programmes: tools used and programme director satisfaction. Med Educ. 2008;42(9):879-86.

2. Fluit C, Bolhuis S, Grol R, Ham M, Feskens R, Laan R, et al. Evaluation and feedback for effective clinical teaching in postgraduate medical education: Validation of an assessment instrument incorporating the CanMEDS roles. Medical Teacher. 2012;34(11):893-901.

3. Yee A, Adjei N, Ford M, Finkelstein J. Do patient expectations of spinal surgen relate to functional outcome? Clinical Orthopaedics and Related Research. 2008:466(5):1154-61.

4. Ware Jr JE, The SCD, MOS. 36-item short-form health survey (SF-36). I. Conceptual framework and item selection. Med Care. 1992;30(6):473-83.

5. QSR NVIVO 10 [computer program]. Doncaster, Victoria, Australia: QSR International Pty Ltd.; 2013

6. Landis JR, Koch GG. The measurement of observer agreement for categorical data. Biometrics. 1977;33(1):159-74.
7. Stata [computer program]. Version 13. 4905 Lakeway Drive, College Station, TX 778452013.

8. Hayes B, Solyom CAE, Wing PC, Berkowitz J. Use of psychometric measures and nonorganic signs testing in detecting nomogenic disorders in low back pain patients. Spine. 1993;18(10):1254-62

9. Waddell G, McCulloch JA, Kummel E, Venner RM. Nonorganic physical signs in low-back pain. SPINE. 1980:5(2):117-25.

\section{Submit your next manuscript to BioMed Central and take full advantage of:}

- Convenient online submission

- Thorough peer review

- No space constraints or color figure charges

- Immediate publication on acceptance

- Inclusion in PubMed, CAS, Scopus and Google Scholar

- Research which is freely available for redistribution

Submit your manuscript at www.biomedcentral.com/submit
C Biomed Central 